\title{
Live images of RNA polymerase II transcription units
}

\author{
Snehal Patel ${ }^{1}$, Natalya Novikova ${ }^{2}$, Brent Beenders ${ }^{2}$, Christopher Austin ${ }^{2} \&$ Michel Bellini $^{2 *}$ \\ ${ }^{1}$ Department of Biochemistry and College of Medicine, School of Molecular and Cellular Biology, University \\ of Illinois at Urbana-Champaign, Urbana, IL 61801, USA; ${ }^{2}$ Department of Cell and Developmental Biology, \\ School of Molecular and Cellular Biology, University of Illinois at Urbana-Champaign, Urbana, IL 61801, USA; \\ Tel: 217-265-5297; Fax: 217-244-6418; E-mail: bellini@life.uiuc.edu \\ * Correspondence
}

Received 2 November 2007. Received in revised form and accepted for publication by Herbert Mcgregor 14 November 2007

Key words: germinal vesicle, lampbrush chromosome, oocyte, transcription unit, Xenopus laevis

\begin{abstract}
The nucleus of an amphibian oocyte can be manually isolated in mineral oil where it maintains all its activities for several hours. These undisrupted (live) nuclei have been used successfully in recent years to analyze the dynamic organization of several non-chromosomal nuclear organelles. Here, we describe an improved procedure for imaging an oil-isolated nucleus by light microscopy and we use it to produce the very first images of lampbrush chromosomes in an in vivo-like condition. These chromosomes are morphologically identical to those observed in conventional nuclear spread preparations. Importantly, their lateral loops, which are active RNA polymerase II transcription units, are readily distinguished by differential interference contrast microscopy.
\end{abstract}

\section{Abbreviations}

AMD

DIC

IGC

LBC

MCD1

RNAPII

YFP

\section{Introduction}

Chromatin takes center stage today as perhaps one of the most complex structures in the cell, being capable of extraordinary conformational plasticity and dynamic exchanges with its surrounding nucleoplasm to orchestrate a variety of fundamental processes such as DNA replication and regulation of gene expression. In cultured somatic cells, efforts to monitor sites of transcription are hindered by the dense grouping of somatic nuclear structures and the low resolution of light microscopy. In contrast to somatic nuclei, the nucleus of the amphibian oocyte, also named the germinal vesicle $(\mathrm{GV})$, offers the unique opportunity to study RNA transcription and processing with a high spatial resolution.

The lampbrush chromosomes (LBCs) of the oocyte are extended diplotene bivalent chromosomes in which the very high transcription activity of RNA polymerase II (RNAPII) results in the presence of numerous lateral loops along the length of each homologue (excellently reviewed in Morgan 2002). Each chromosomal loop corresponds to a DNA axis actively transcribed by RNAPII and surrounded by tightly packed nascent RNP fibrils. The standard preparation of nuclear spreads to visualize LBCs, however, results in a complete loss of the nucleoplasm and, thus, prevents in vivo studies of the LBCs and associated loops. In addition, high-resolution imaging of the nucleus in live oocytes has been prevented until recently by the large size of the oocyte $(0.8-1.2 \mathrm{~mm})$ and the 
presence of a high concentration of yolk and pigment granules in the cytoplasm.

The breakthrough came with the simple yet effective finding that nuclei isolated in mineral oil can be flattened between a microscope slide and a cover glass to permit a direct in vivo observation of the nuclear organelles by light microscopy (Handwerger et al. 2003). Oil-isolated nuclei maintain their structures and functions for many hours (Lund \& Paine 1990, Paine et al. 1992) and were used to study the steadystate dynamics of several components of the Cajal body (Handwerger et al. 2003, Deryusheva \& Gall 2004, Gall et al. 2004), a nuclear organelle implicated in the transcription and processing of all nuclear RNAs (reviewed in Gall 2003). Furthermore, the physical structure of Cajal bodies and two other organelles, nucleoli and interchromatin granule clusters (IGCs), were also analyzed (Handwerger et al. 2005). While all the nuclear organelles were readily identifiable, LBCs remained elusive.

Here, we show that the apparent lack of LBCs from oil-isolated nuclei is due to their extensive damage during sample preparation. We also detail an improved preparation method that preserves chromosomal integrity, and we use it to present the very first images of LBCs in intact, unfixed nuclei. We show that these chromosomes are morphologically identical to those observed in nuclear spread preparations. Remarkably, their lateral loops are readily observable by difference interference contrast (DIC) microscopy, which represents the very first visualization of RNAPII transcription units in a live nucleus.

\section{Material and methods}

Oocytes, microinjections and nuclear spread

Fragments of ovary were surgically removed from adult female frogs (Xenopus laevis) anesthetized in
$0.15 \%$ tricaine methane sulfonate (MS222, Sigma Chemical, St. Louis, MO, USA). Stage IV-VI oocytes were manually separated using fine tweezers and maintained in saline buffer OR2 (Wallace et al. $1973)$ at $18^{\circ} \mathrm{C}$. In some experiments, actinomycin D (Sigma) was used at $10 \mu \mathrm{g} / \mathrm{ml}$ in OR2 to inhibit RNA transcription. Nuclear spreads were prepared as described previously in Patel et al. (2007). After fixation, nuclear spreads were rinsed in PBS and mounted in $50 \%$ glycerol containing $1 \mathrm{mg} / \mathrm{ml}$ phenylenediamine and $10 \mathrm{pg} / \mathrm{ml}$ 4,6-diamidino-2phenylindole (DAPI). DAPI was also used in oilisolated nuclei to identify LBCs. In this case, oocytes were injected into the cytoplasm (20 $\mathrm{nl}$ of a $5 \mathrm{ng} / \mu \mathrm{l}$ solution of DAPI in water). Glass needles were prepared using a horizontal pipette puller P-97 (Sutter Instrument, Novato, CA, USA). All injections were performed using a nanojet II (Drummond, Broomal, PA, USA) under an S6 Leica dissecting microscope (Heidelberg, Germany).

\section{Oil-isolation of nuclei and preparation for microscopy}

The isolation of nuclei in mineral oil (Sigma) is performed as described in Paine et al. (1992). When needed, isolated nuclei were transferred into a small oil-containing plastic Petri dish and maintained at $18^{\circ} \mathrm{C}$. The wax spacer needed for imaging LBCs is produced directly onto microscope slides using the following method: $20 \mathrm{mg}$ of a 1:1 mixture of petroleum-paraffin wax is melted onto a microscope slide under a $25 \mathrm{~mm}$ acrylic square containing a circular hole ( $5 \mathrm{~mm}$ in diameter) in its center, using a hotplate $\left(\sim 80^{\circ} \mathrm{C}\right)$. After the homogeneous spreading of the melted wax between the acrylic square and the slide, the wax is allowed to re-solidify by letting the slide cool slowly. Once the wax has hardened (5 min.), the acrylic square is removed using a razor blade and a thin layer of wax $(\sim 25-30 \mu \mathrm{m}$ in

Figure 1. The oil-isolation procedure does not affect chromosome architecture. A Phase-contrast micrograph showing one disrupted LBC within a small nucleoplasmic region of an oil-isolated nucleus. The chromosomal axes display an abnormal structure that consists of extensively stretched chromatin fibers (arrows) interspaced with small regions of more condensed chromatin (arrowheads). Note that lateral loops are absent. The nucleus was prepared using the method described in Handwerger et al. (2003), which is also schematically represented here. Two Cajal bodies $(*)$ are readily distinguished from the other organelles. B A differential interference contrast (DIC) image showing one LBC surrounded by several organelles on a nuclear spread. As indicated schematically, the nucleus was isolated and maintained in mineral oil for $5 \mathrm{~h}$ before transferring it to the saline buffer for nuclear spread preparation. Both LBCs and organelles were found to exhibit normal structures. In particular, chromosomes displayed numerous lateral loops. Both chromosomal axes as well as Nucleoli were well labeled with DAPI. Notice that chromosomal loops, IGCs, and CBs are weakly labeled (most likely because of their high RNA content). Scale bar is $10 \mu \mathrm{m}$. 
thickness) with a hole in its center is left imprinted on the slide. An oil-isolated nucleus can then be deposited together with $\sim 8 \mu$ l of oil in the central shallow well. Finally, an $18 \mathrm{~mm}$ square coverslip (Corning Inc., Corning, NY, USA) is apposed gently onto the specimen, which is then ready for microscopy.
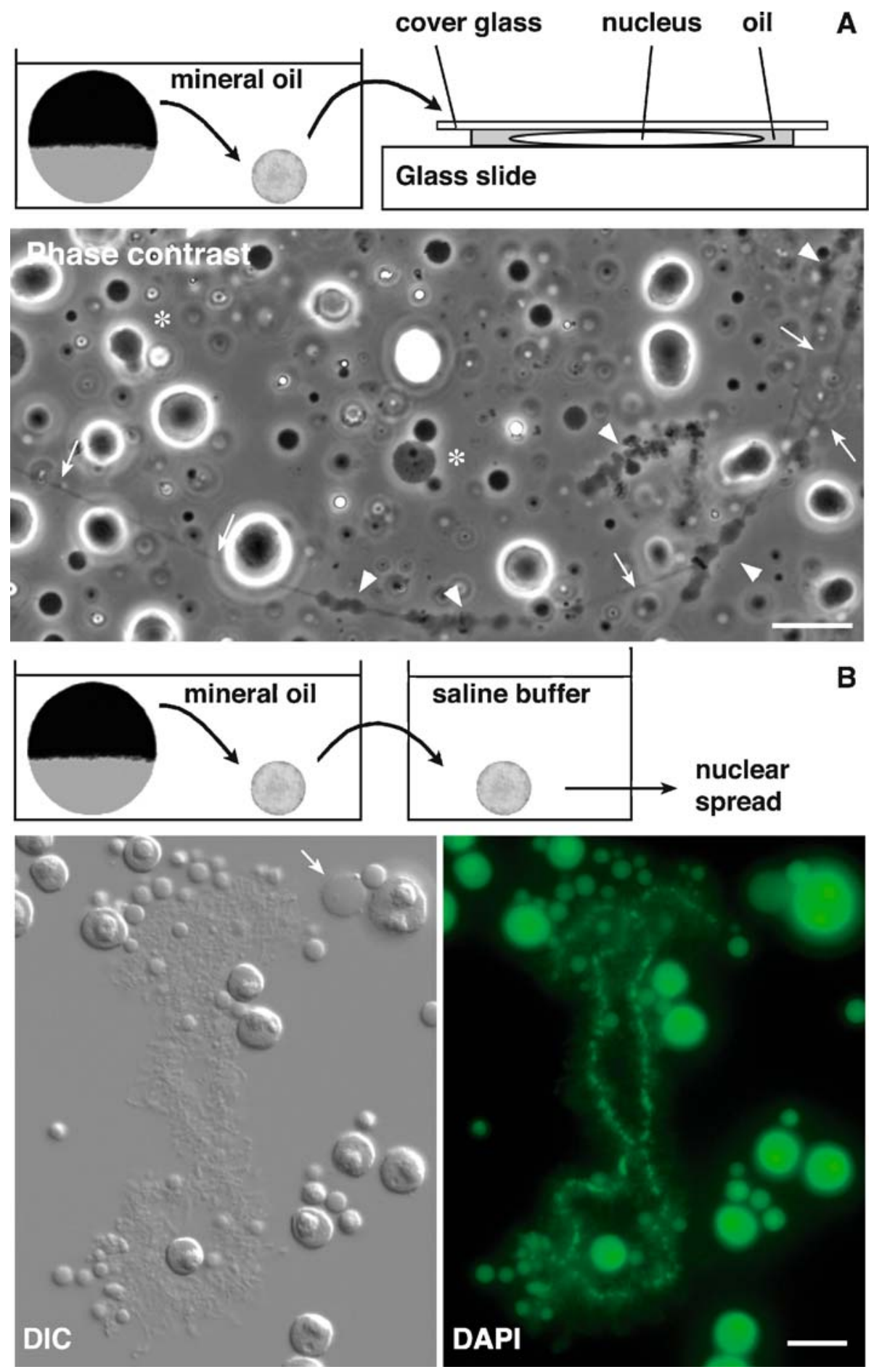
Transferring a nucleus from mineral oil to an aqueous saline buffer

The method was developed by Dr. Joseph Gall and is as follows. A small drop of oil is deposited at the bottom of a plastic Petri dish containing the 5:1 buffer $(83 \mathrm{mM} \mathrm{KCl}, 17 \mathrm{mM} \mathrm{NaCl}, 6.5 \mathrm{mM}$ $\mathrm{Na}_{2} \mathrm{HPO}_{4}, 3.5 \mathrm{mM} \mathrm{KH} \mathrm{PO}_{4}, 1 \mathrm{mM} \mathrm{MgCl} 2,1 \mathrm{mM}$ DTT) where cohesive forces maintain it in place. Oilisolated nuclei are then directly pipetted in the oil drop. Using fine tweezers, nuclei are pushed one by one to the oil-buffer interface. The first nucleus is totally disrupted, presumably because of tension at the surface of the oil drop. Its content is not lost in the buffer, however, but rather appears to coat the surface of the oil drop. Importantly, such a conditioned surface lets the other nuclei pass intact from oil to the aqueous buffer. Nuclear spreads can then be prepared as in Patel et al. (2007).

\section{Microscopy}

All microscopy was performed on an upright Leica DMR (Heidelberg, Germany). Standard fluorescence microscopy was carried out using a PL Fluotar 40X oil objective (NA=1.0) and a HCL FL Fluotar 100× oil objective (NA=1.30). Images were captured using a monochrome Retiga EXI Charge-Coupled Device (CCD) camera (Qimaging, Surrey, BC, Canada) driven by the In vivo software (version 3.2.0, Media Cybernetics, Bethesda, MD, USA). All images were captured at room temperature. Figures were processed using Adobe Photoshop CS version 8.0 and assembled with Adobe InDesign CS version 3.0.

Fluorescence recovery after photobleaching (FRAP) was performed using the SRS NL100 MicroPoint Laser System (Photonic Instruments Inc., St. Charles, IL, USA) adapted to our DMR microscope. A $514 \mathrm{~nm}$ laser dye was used for the photobleaching of yellow fluorescent protein (YFP). Data acquisition was done using the In vivo software (version 3.2.0, Media Cybernetics). The protein YFP-MCD1 was expressed by microinjection of its corresponding transcript using a strategy described in Patel et al. (2007).

\section{Results}

The isolation of nuclei in oil does not modify nuclear structure

When oil-isolated nuclei are directly flattened under a cover glass for observation by light microscopy as described by Handwerger et al. (2003), organelles such as Cajal bodies, nucleoli and IGCs display normal morphology (Figure 1). In contrast, we find that LBCs are difficult to observe because of extensive physical damage. Indeed, LBCs are stretched beyond their elasticity limit, resulting in many breaks and the anomalous presence of very thin chromatin fibers (Figure 1A), which can be labeled with DNA dyes such as DAPI. We further demonstrate that this dramatic structural change is due to the apposition of the cover glass rather than the isolation of the nucleus in mineral oil itself. Oilisolated nuclei from stage $\mathrm{V}$ oocytes were left in oil at $18^{\circ} \mathrm{C}$ for several hours and subsequently recovered into a physiological buffer for nuclear spread preparations (see Material and methods). Figure 1B shows that LBCs from these nuclei display a normal architecture (reviewed in Morgan 2002). In particular, the lateral loops are readily distinguishable over the entire length of both homologues, and there is no obvious alteration of the chromosomal axes, highlighted here by DAPI staining.

\section{Visualization of LBCs in oil-isolated nuclei}

These findings prompted us to alter the flattening step during sample preparation to prevent mechanical disruption of the LBCs. We find that a very effective modification is the insertion of a spacer of $\sim 20 \mu \mathrm{m}$ between the microscope slide and the cover glass (see Material and methods) as shown schematically in

Figure 2. Lampbrush chromosomes in oil-isolated nuclei. A Fluorescent micrographs of oil-isolated nuclei from stage IV and VI oocytes. As indicated schematically, oocytes were injected with DAPI $(0.1 \mathrm{ng})$ before nuclear isolation. In agreement with their respective transcriptional activities, stage IV oocytes have LBCs that are consistently more extended than the one from stage VI oocytes. At high concentration, such as $20 \mathrm{ng} /$ oocyte, DAPI inhibits transcription efficiently, which results in very condensed LBCs. Scale bar is $10 \mu \mathrm{m}$. B Magnified views of chromosomal axes are presented together with their corresponding DIC images. Stage IV axes are visible by DIC primarily because of the numerous lateral loops. In contrast, stage VI axes appear as linear arrays of condensed chromatin domain from which fewer/smaller loops are projected. Arrows indicate several large loops. DAPI labeling was pseudo-colored in green. Scale bar is $5 \mu \mathrm{m}$. 


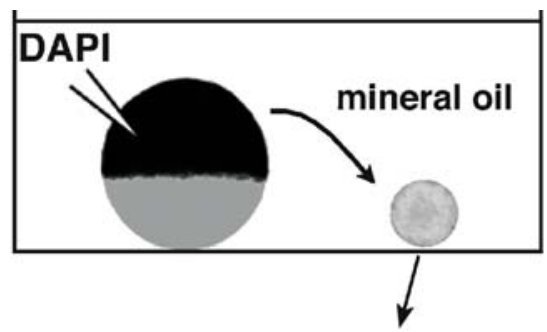

cover glass nucleus mineral oil

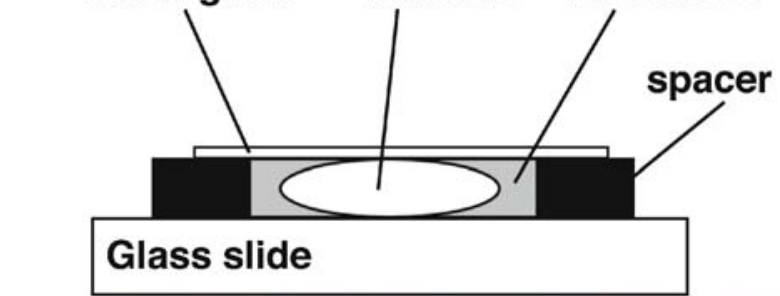

Glass slide

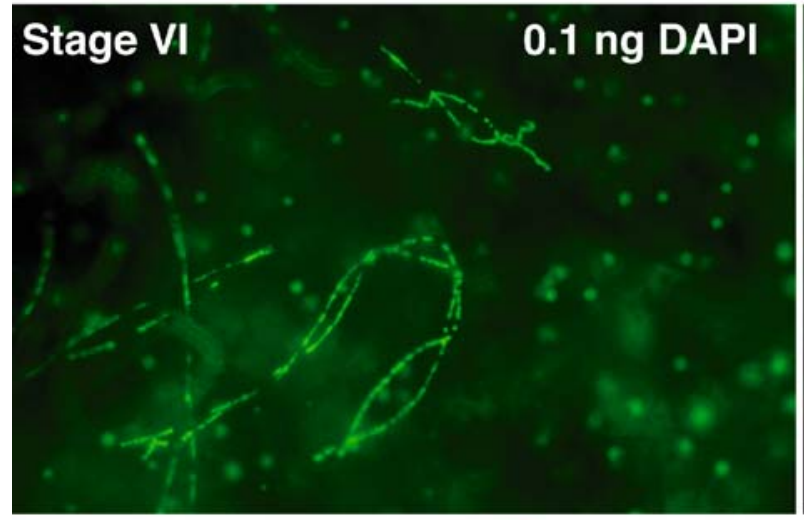

Stage IV

$0.1 \mathrm{ng}$ DAPI

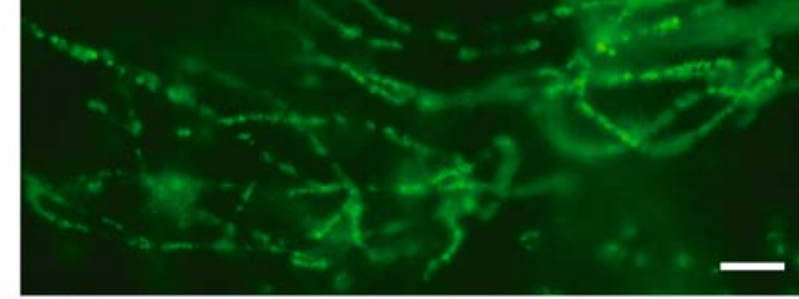

Stage IV

20 ng DAPI
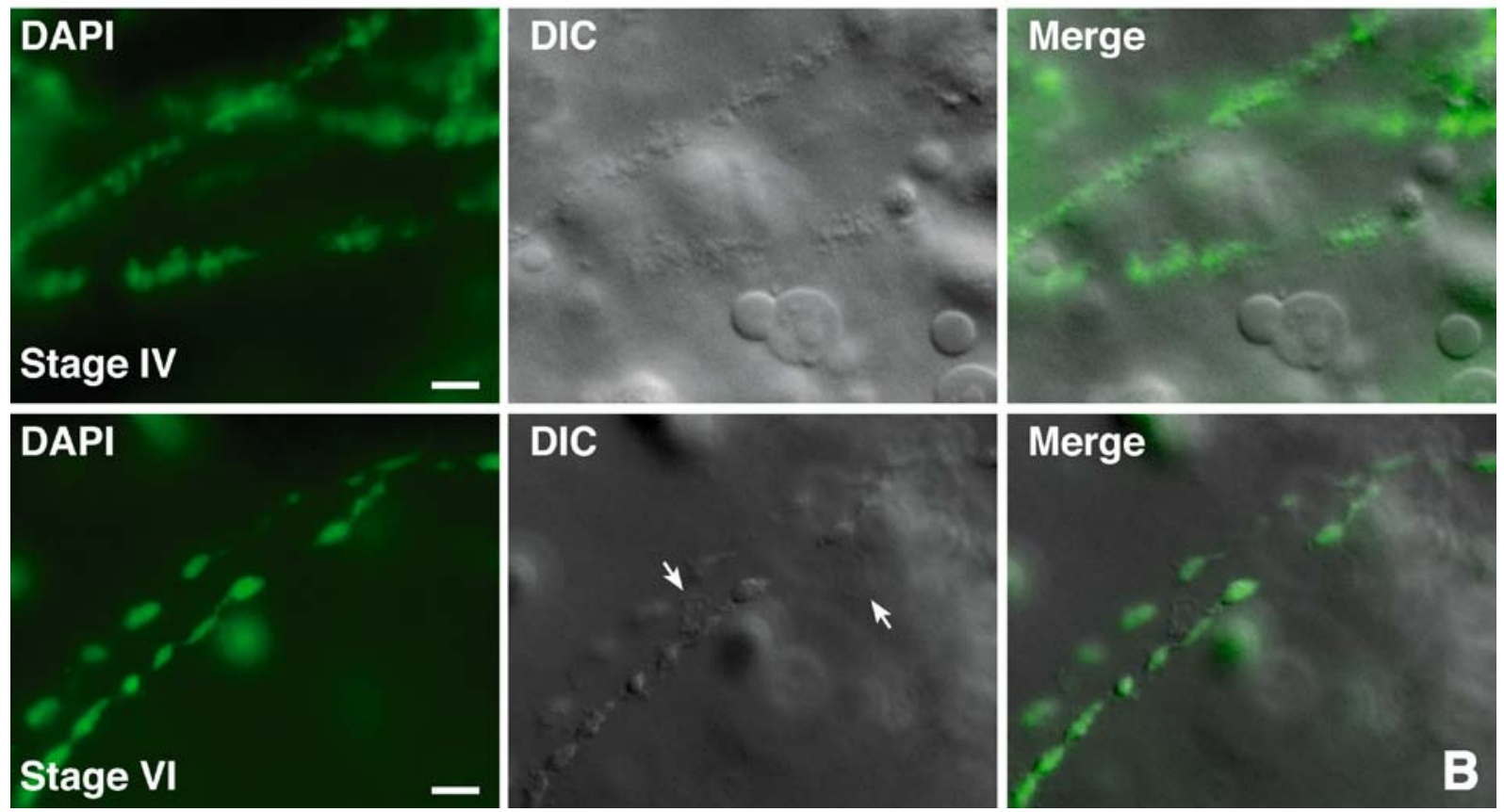
Figure 2A. Stages IV and VI oocytes were injected with $100 \mathrm{pg}$ of DAPI in water, and nuclei were isolated in mineral oil 30 minutes later. In all cases, chromosomes could readily be identified as bivalent structures using fluorescence microscopy (Figure 2A and B). LBCs from stage IV oocytes were considerably more extended than the LBCs from stage VI oocytes. This difference in chromatin organization is correlated with the reduced transcriptional activity of the nucleus within the late stages of oogenesis and is also observed on conventional nuclear spreads. We also observed that a higher concentration of DAPI such as $20 \mathrm{ng} /$ oocyte has a dramatic effect on the physiology of the chromosomes. Under this condition, LBCs appear very condensed (Figure 2A), and the absence of chromosomal loops indicates that RNA transcription is totally inhibited. While it is not clear how DAPI influences LBC architecture, its effect does not depend on UV irradiation and occurs within minutes after injection (our unpublished data). The amount of $100 \mathrm{pg}$ of DAPI/oocyte was thus determined experimentally to permit a rapid identification of LBCs in
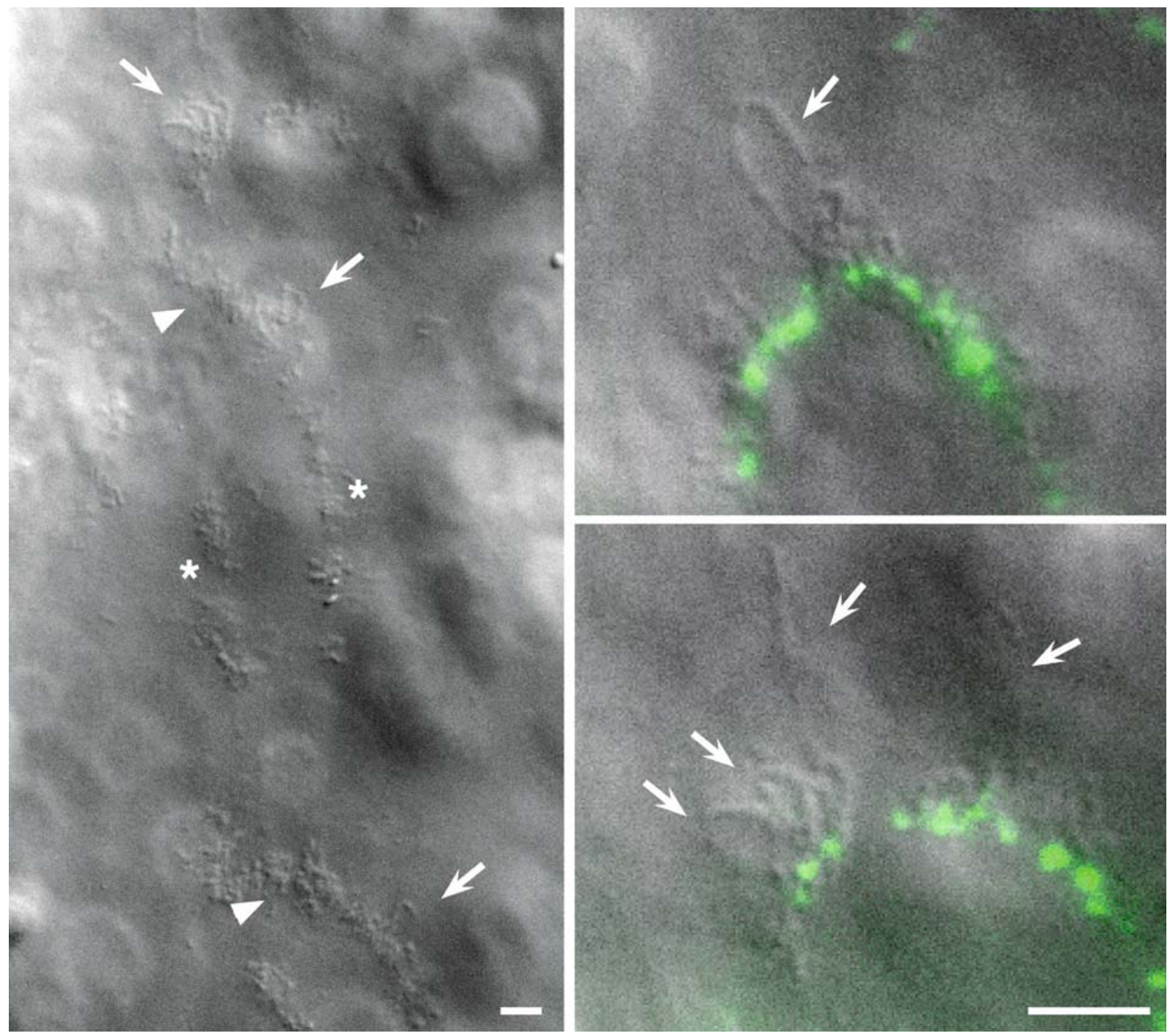

Figure 3. DIC images of individual RNAPII transcription units in oil-isolated nuclei. DIC micrograph of an LBC in oil-isolated nucleus from a stage IV oocyte (left panel). The two homologues (indicated by *) are still paired at chiasmata (arrowheads). Arrows indicate particularly extended loops. Magnified views of chosen sets of loops are also presented. There, DAPI staining is merged (pseudo-colored in green) to indicate the position of chromosomal axes. Scale bars are $5 \mu \mathrm{m}$. 
oil-isolated nuclei without any obvious effect on their physiology. Surprisingly, LBCs are consistently found all grouped within one limited region of the large nucleoplasmic volume. This remarkable organization suggests that LBCs are not free to diffuse away from each other.

\section{RNA polymerase II transcription units}

One of the most obvious characteristics of LBCs on nuclear spreads is the presence of numerous lateral loops, which are active RNAPII transcription units.
We are pleased to report that the same organization is exhibited by LBCs in oil-isolated nuclei. The DIC image of one such LBC is presented in Figure 3. The two homologues, still attached at chiasmata (indicated by arrowheads), are readily distinguished from the nucleoplasm. Importantly, numerous loops are projected away from each chromosomal axis. Unlike in nuclear spread preparations, however, the loops are not brought into the two-dimensional plane of the microscope slide surface by centrifugation. Rather the loops are distributed radially from the chromosomal axes, which makes it

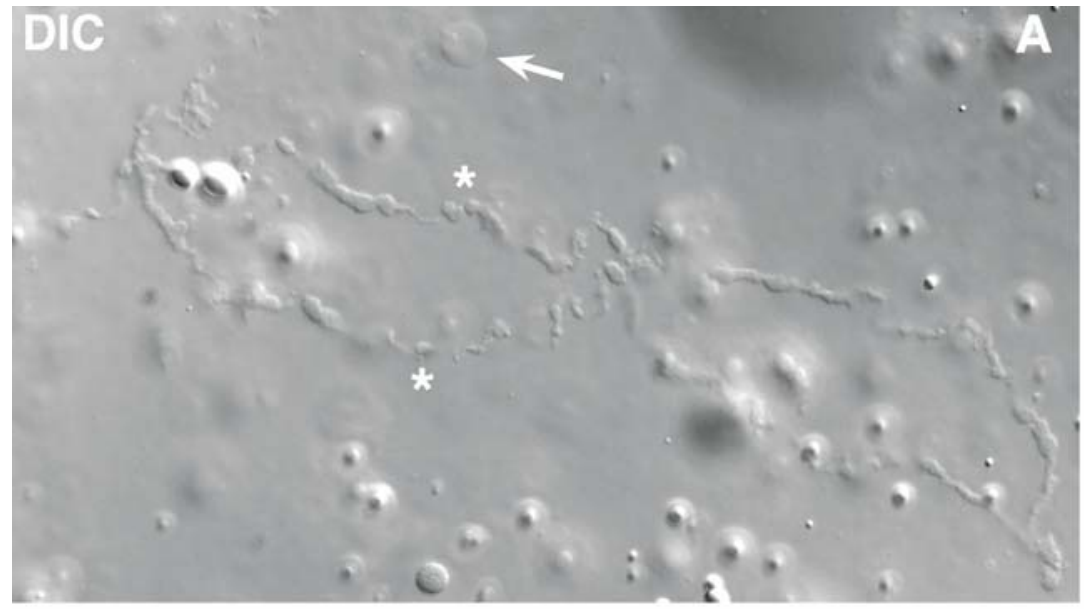

\section{DAPI}
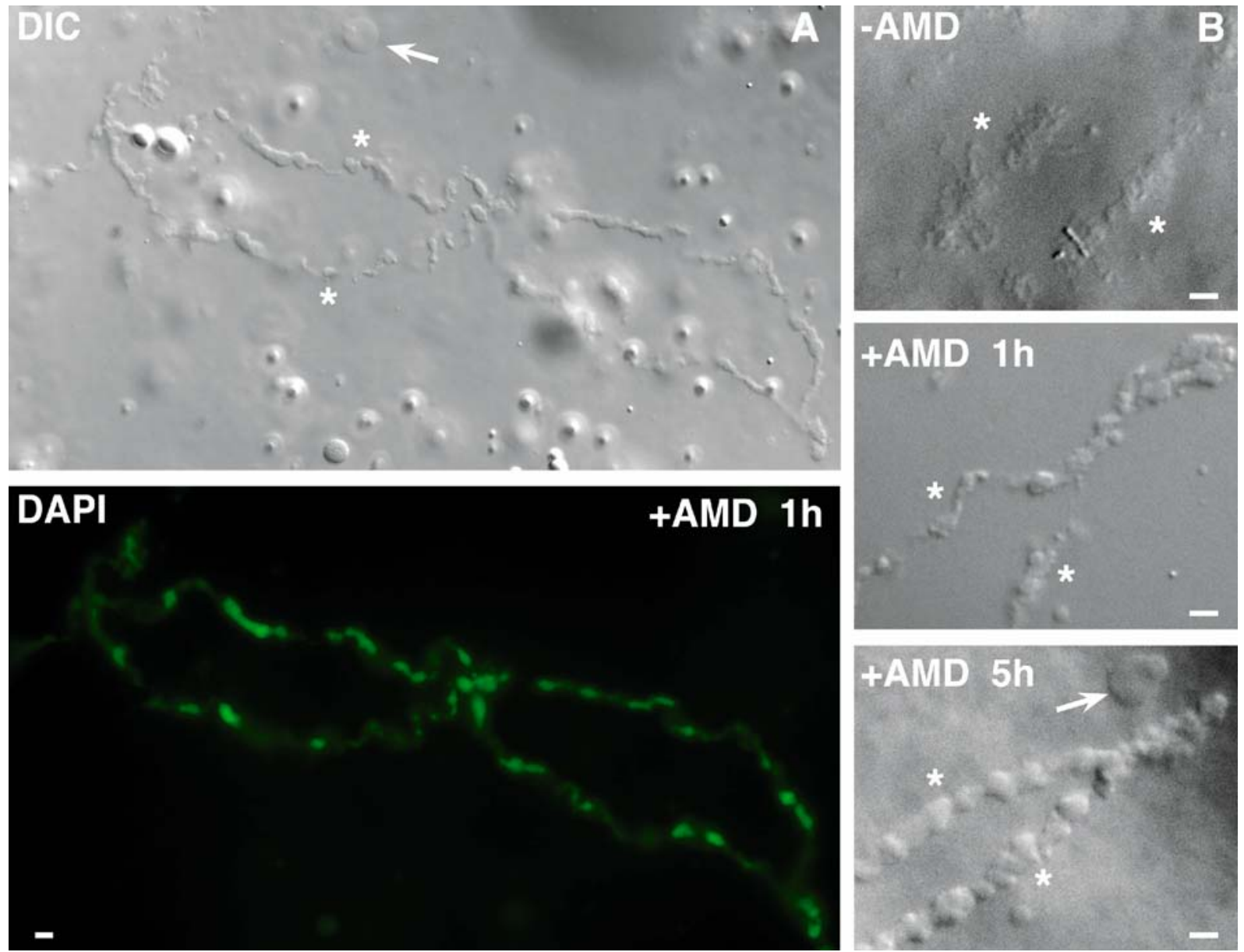

Figure 4. LBC structure in oil-isolated nuclei after actinomycin D treatment. A DIC micrograph and its corresponding fluorescent image (DAPI) of one LBC in a live nucleus. A stage IV oocyte was treated with actinomycin D (AMD) for $1 \mathrm{~h}$ before the isolation of its nucleus in mineral oil. Chromosomal axes, which are well labeled with DAPI, are also readily identified by DIC. As expected, the lateral loops, which are active sites of transcription for RNAPII, are absent. B DIC images showing the effect of AMD over time on the architecture of LBCs. Only a small chromosomal region is shown in each panel. LBCs from control stage IV oocytes display extended axes, organized in repeats of loop clusters (-AMD). Inhibition of transcription by AMD rapidly (+AMD $1 \mathrm{~h}$ ) results in the loss of the lateral loops and a thickening of the chromosomal axes. Over time (+AMD $5 \mathrm{~h}$ ), chromosomes shorten and the axes appear as linear arrays of tightly packed chromatin domains. In all images, the two homologues of the same LBC are indicated by *. Arrows indicate CBs. Scale bars are $5 \mu \mathrm{m}$. 
difficult to image them all in the same focal plan. A magnified view of two sets of loops is also presented in Figure 3 to further demonstrate that the bases of these loops are tethered to a chromosomal axis, which is defined here using DAPI fluorescence. We then tested whether these loops were sensitive to the transcription inhibitor actinomycin D (AMD). It is well known from previous studies using nuclear spread preparations that treatment of oocytes with AMD, or other transcriptional inhibitors, induces a dramatic retraction of the loops and an overall condensation of LBCs. Figure 4 shows LBCs of oil-isolated nuclei from AMD-treated oocytes. After $1 \mathrm{~h}$ of treatment,

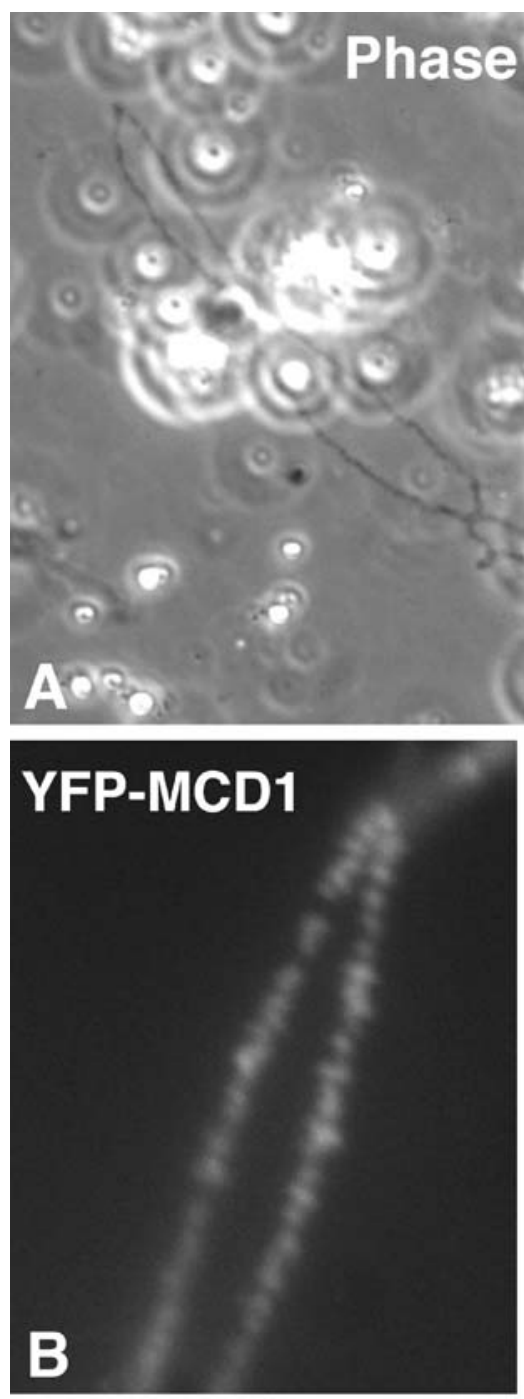

Pre-bleaching

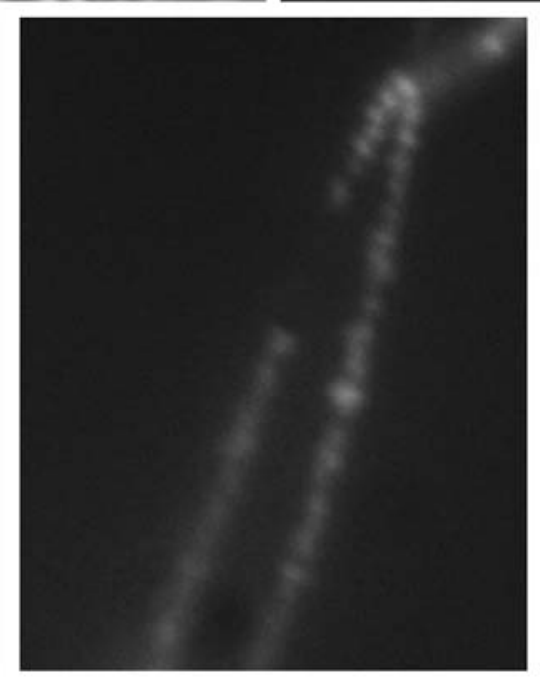

Post-bleaching

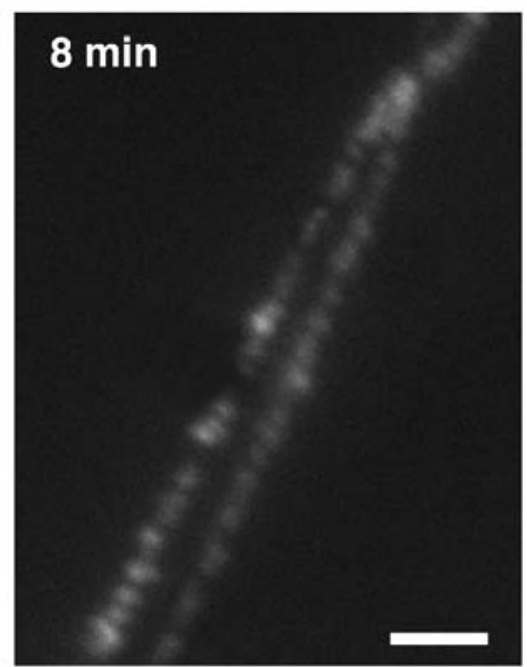

after recovery

Figure 5. Steady-state dynamics of chromosomal YFP-MCD1. A A phase-contrast image and its corresponding fluorescent micrograph showing the chromosomal distribution of the newly expressed YFP-MCD1 in an oil-isolated nucleus from a stage VI oocyte. As expected for a member of the cohesin complex, MCD1 associates with chromosomal axes where sister chromatid cohesion is occurring. Scale bar is $10 \mu \mathrm{m}$. B Fluorescence bleaching and recovery of chromosomal YFP-MCD1. A narrow region of one of the two homologues forming an LBC was photobleached ("post-bleaching" panel) and recovery of fluorescence was monitored over time. Scale bar is $5 \mu \mathrm{m}$. 
LBCs still display an extended shape but, unlike in control oocytes, the lateral loops are missing. With longer times of exposure to the AMD treatment, LBCs shorten and chromosomal axes are now shaped into linear arrays of immediately adjacent condensed chromatin domains.

In summary, it is now possible to study LBCs in an isolated and fully functional nucleus where their overall dynamic architecture is essentially identical to that previously defined on nuclear spreads (see Morgan 2002 for review).

\section{Toward the study of steady-state dynamics} of LBC components

The highly extended form of LBCs and the very large nucleoplasmic volume of the oocyte nucleus permit chromatin structures to be distinguished from the nucleoplasm and other nuclear structures unambiguously using a light microscope. Oil-isolated nuclei thus represent a powerful system to study the in vivo steady-state dynamics of chromosomal factors. Figure 5 is presented here to demonstrate that it is now possible to monitor the dynamic exchanges of a chromosomal factor between $\mathrm{LBCs}$ and the nucleoplasm. The protein MCD1 (mitotic chromosome determinant 1) is a subunit of the cohesin complex, which regulates sister chromatid cohesion (reviewed in Guacci 2007). When expressed in fusion with YFP in stage IV-VI oocytes, YFPMCD1 associates specifically with chromosomal axes (each formed of two paired sister chromatids) where it co-localizes with other members of the cohesin complex (our unpublished data). The fluorescence recovery of YFP-MCD1 on chromosomal axes after photobleaching is presented in Figure 5. The fact that the fluorescent signal was recovered within minutes highlights the dynamic behavior of chromosomal MCD1.

\section{Discussion}

\section{A renewal of the lampbrush chromosome?}

Every major cell biology textbook, such as Molecular Biology of the Cell (Alberts et al. 2002), shows a micrograph of LBCs in support of the well-accepted hypothesis that somatic chromatin organizes into looped domains in all eukaryotes. LBCs were also used in early chromosome-stretching experiments (Callan 1955) to pioneer the idea that they are extensible and elastic structures. Finally, DNAse treatments of LBCs were used to demonstrate that each chromatid contains a single DNA molecule (Callan \& Macgregor 1958, Gall 1963). Despite such a distinguished legacy, very little work is currently being done using LBCs to tackle current questions on chromatin organization. Here, we present a new method for visualizing LBCs in oil-isolated nuclei, which we hope will renew interest in using them to study fundamental aspects of chromatin dynamics in vivo. We find that in such nuclei LBCs display the overall architecture of actively transcribing bivalent chromosomes, as seen on nuclear spreads. Such organization was expected since it was previously shown that an oil-isolated nucleus maintains all its activities for several hours (Paine et al. 1992). In particular, transcription is still very active and accordingly we observe the presence of numerous chromosomal loops that are sensitive to AMD. Importantly, our data demonstrate that the conventional method for nuclear spread preparation does not alter LBC physiology.

While DAPI is used here to rapidly identify LBCs within the large nucleoplasmic volume of an oocyte nucleus, it is not required. Indeed, with practice one can distinguish LBCs from other nuclear structures simply using DIC. Another strategy to unambiguously identify chromosomal axes without compromising the functional integrity of LBCs is to induce the expression of a fluorescently tagged chromosomal protein in oocytes prior the isolation of nuclei. A good example is provided in Figure 5 by the protein YFP-MCD1.

LBC clusters?

In oil-isolated nuclei, LBCs are very often found all grouped into a limited nuclear domain. Interestingly, a similar aggregation of LBCs in the middle of the nucleus was previously reported in specific developmental stages of the axolotl oocyte (Callan 1966). While it is not clear why LBCs do not distribute throughout the nucleoplasmic volume, it suggests the interesting possibility that they are tethered among each other. Over the years, evidence that DNAcontaining filaments connect mitotic chromosomes 
has been obtained (see Poirier \& Marko 2002, 2003 for reviews), and it was proposed recently that satellite DNA-containing bridges could link metaphase chromosomes (Kuznetsova et al. 2007). Whether similar chromatin bridges tether the meiotic diplotene LBCs together is an interesting question that will require further investigation.

\section{Studying functional LBCs}

The fact that LBCs can be observed in an oil-isolated nucleus, an essentially in vivo condition, represents a unique opportunity to study chromosome structure and function. We present here a brief qualitative FRAP analysis of the chromosomal YFP-MCD1 dynamics as an example. It is important to note that the bleached region can be precisely defined. In this case, only a small domain of one of the two homologous axes was photobleached. The other axis conveniently served as an internal control to monitor changes of fluorescence signals due to data acquisition. Such a level of spatial resolution simply cannot be achieved with any other system. Because the chromosomal loops are also easily distinguishable in oil-isolated nuclei, one of the most exciting future developments of the present work will be to use similar approaches to that used for MCD1 to tackle fundamental questions regarding the dynamic organization of the transcription unit.

\section{Acknowledgments}

We are very grateful to Drs. Joseph Gall (Carnegie Institution of Washington), Herbert Macgregor (University of Exeter), Robert Clegg (UIUC) and John Sedat (UCSF) for very productive discussions and encouragements. We thank Dr. Guacci (Carnegie Institution of Washington) for the MCD1 cDNA clone. We would also like to acknowledge the reviewers for their insightful comments. This work was supported by a CAREER Award from the National Science Foundation.

\section{References}

Alberts B, Johnson A, Lewis J, Raff M, Roberts K, Walter P (2002) Molecular Biology of the Cell. New York: Garland Science, Taylor and Francis group, pp. 117-118.

Callan HG (1955) Recent work on the structure of cell nuclei. In: Fine Structure of Cells: Symposium of the VIIIth Congress in Cell Biology, Leiden 1954, Vol. 21. Groningen: Noordhof, pp. 89-109.

Callan HG (1966) Chromosomes and nucleoli of the axolotl, Ambystoma mexicanum. J Cell Sci 1: 85-108.

Callan HG, Macgregor HC (1958) Action of deoxyribonuclease on lampbrush chromosomes. Nature 181: 1479-1480.

Deryusheva S, Gall JG (2004) Dynamics of coilin in Cajal bodies of the Xenopus germinal vesicle. Proc Natl Acad Sci USA 101: $4810-4814$.

Gall JG (1963) Kinetics of deoxyribonuclease action on chromosomes. Nature 198: 36-38.

Gall JG (2003) The centennial of the Cajal body. Nat Rev Mol Cell Biol 4: 975-980.

Gall JG, Wu Z, Murphy C, Gao H (2004) Structure in the amphibian germinal vesicle. Exp Cell Res 296: 28-34.

Guacci V (2007) Sister chromatid cohesion: the cohesin cleavage model does not ring true. Genes Cells 12: 693-708.

Handwerger KE, Murphy C, Gall JG (2003) Steady-state dynamics of Cajal body components in the Xenopus germinal vesicle. $J$ Cell Biol 160: 495-504.

Handwerger KE, Cordero JA, Gall JG (2005) Cajal bodies, nucleoli, and speckles in the Xenopus oocyte nucleus have a low-density, sponge-like structure. Mol Biol Cell 16: 202-211.

Kuznetsova IS, Enukashvily NI, Noniashvili EM (2007) Evidence for the existence of satellite DNA-containing connection between metaphase chromosomes. J Cell Biochem 101: 1046-1061.

Lund E, Paine PL (1990) Nonaqueous isolation of transcriptionally active nuclei from Xenopus oocytes. Methods Enzymol 181: 36-43.

Morgan GT (2002) Lampbrush chromosomes and associated bodies: new insights into principles of nuclear structure and function. Chromosome Res 10: 177-200.

Paine PL, Johnson ME, Lau Y-T, Tluczek LJM, Miller DS (1992) The oocyte nucleus isolated in oil retains in vivo structure and functions. Biotechniques 13: 238-245.

Patel SB, Novikova N, Bellini M (2007) Splicing-independent recruitment of spliceosomal small nuclear RNPs to nascent RNA polymerase II transcripts. J Cell Biol 178: 937-949.

Poirier MG, Marko JF (2002) Micromechanical studies of mitotic chromosomes. J Muscle Res Cell Motil 23: 409-431.

Poirier MG, Marko JF (2003) Micromechanical studies of mitotic chromosomes. Curr Top Dev Biol 55: 75-141.

Wallace RA, Jared DW, Dumont JN, Sega MW (1973) Protein incorporation by isolated amphibian oocytes: III. Optimum incubation conditions. J Exp Zool 184: 321-333. 